\title{
Diagnóstico y tratamiento precoz de la hipoacusia unilateral o asimétrica en la infancia: recomendaciones CODEPEH 2017
}

\section{Early diagnosis and treatment of unilateral or asymmetrical hearing loss in childhood: 2017 CODEPEH recommendations}

\section{Palabras clave}

Hipoacusia unilateral, hipoacusia asimétrica, audición binaural, procesamiento auditivo, calidad de vida, discapacidad auditiva.

\section{Keywords}

Unilateral hearing loss, asymmetrical hearing loss, binaural hearing, hearing processing, quality of life, hearing disability.

\section{Introducción ${ }^{1}$}

El término "hipoacusia unilateral" $(\mathrm{HU})$ se define por la presencia de una pérdida de audición en uno de los dos oídos, siempre que el umbral sea superior a $25 \mathrm{~dB}$ en todas las frecuencias. El término "hipoacusia bilateral asimétrica" (HA) se refiere a aquella hipoacusia bilateral con umbrales auditivos de $25 \mathrm{~dB}$ o superiores en el mejor oído en todas las frecuencias (Lin et al., 2017: 912919). Se ha de constatar una diferencia entre los umbrales de ambos oídos mayor de $15 \mathrm{~dB}$ en las frecuencias 500,1000 y $2000 \mathrm{~Hz}$, o mayor de $20 \mathrm{~dB}$ en las frecuencias 3000, 4000 y $6000 \mathrm{~Hz}$ (May, 2000: 112-120).

Existen evidencias de que la presencia de una HU o HA congénita, o adquirida en edades tempranas, puede afectar negativamente al desarrollo del niño, no solamente desde el punto de vista audiológico, sino también en la autoestima y el sobresfuerzo académico necesario.

\footnotetext{
1. El presente documento de Recomendaciones CODEPEH 2017 ha sido elaborado en el marco del proyecto sobre la Detección, diagnóstico y tratamiento precoz de la hipoacusia unilateral o asimétrica en la infancia, que ha sido desarrollado por la Confederación Española de Familias de Personas Sordas-FIAPAS, en colaboración con la Comisión para la Detección Precoz de la Sordera InfantilCODEPEH, con la coorganización del Real Patronato sobre Discapacidad.
}

\section{Faustino Núñez Batalla $<$ fnunezb@uniovi.es>}

Presidente de la CODEPEH. Servicio ORL, Hospital Universitario Central de Asturias-Oviedo. Sociedad Española de Otorrinolaringología

\section{Carmen Jáudenes Casaubón <direccion@fiapas.es>}

Vocal de la CODEPEH. Directora de la Confederación Española de Familias de Personas Sordas (FIAPAS)

\section{José Miguel Sequí Canet}

Vocal de la CODEPEH. Jefe de Servicio de Pediatría, Hospital de Gandía-Valencia. Asociación Española de Pediatría.

\section{Ana Vivanco Allende}

Vocal de la CODEPEH. Servicio de Pediatría, Hospital Universitario Central de Asturias-Oviedo. Asociación Española de Pediatría.

\section{José Zubicaray Ugarteche}

Vocal de la CODEPEH. Servicio ORL Infantil, Complejo Hospitalario de Navarra-Pamplona. Sociedad Española de Otorrinolaringología.

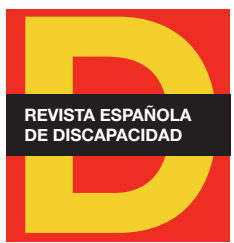

Para citar:

Núñez, F. et al. (2018): "Diagnóstico y tratamiento precoz de la hipoacusia unilateral o asimétrica en la infancia: recomendaciones CODEPEH 2017". Revista Española de Discapacidad, 6 (I): 259-280.

Doi: <https://doi.org/10.5569/23405104.06.01.13>

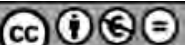


En la actualidad, se está cerca de conseguir la identificación de todos los niños con hipoacusia permanente, incluyendo los casos unilaterales, gracias a los programas de detección precoz.

El despistaje auditivo neonatal ha cambiado enormemente la identificación de los niños con HU. Antes de instituir estos programas de detección precoz neonatal, los niños con $\mathrm{HU}$ se identificaban habitualmente en los programas de cribado escolar. Por tanto, el impacto del despistaje auditivo neonatal en la detección de la HU pediátrica se debe considerar un éxito en salud pública. En un centro terciario académico en Missouri, con anterioridad a la implantación del programa de cribado, el porcentaje de niños con HU neurosensorial detectados antes de los 6 meses de edad era del $3 \%$. Este porcentaje aumentó a un $42 \%$ después de dicha implementación. La edad promedio en el momento del diagnóstico también disminuyó de 4,4 a 2,6 años en este estudio. Sin embargo, la existencia del cribado por sí solo no parece explicar este efecto. Otra revisión retrospectiva de los niños con $\mathrm{HU}$ en un centro académico terciario en Ohio mostró que sólo el $26 \%$ había sido identificado a través del cribado neonatal y la edad media al diagnóstico era todavía 5,6 años. Esto corrobora la aparición tardía de estas sorderas (Vila et al., 2015: 271-278).

La prevalencia de la HU en el recién nacido es de 0,1\% (Lieu, 2015: 1011-1026), aumentando con la edad al 0,27\% debido a los casos de aparición diferida antes de los 5 años y subiendo al 0,35\% en la adolescencia. En la edad escolar se podría encontrar hasta un 3-6\% de niños con distintos grados de HU (Ross et al., 2010: 126-133).

La etiología de la hipoacusia bilateral en los niños ha sido investigada en profundidad y se sabe que más del $50 \%$ de los casos son debidos a causas genéticas, seguidas de infecciones congénitas, ototoxicidad, prematuridad e hipoxia perinatal. En contraste, las causas que provocan la HU y la HA son relativamente menos conocidas: se han encontrado numerosos factores de riesgo como las malformaciones del oído, infección congénita por citomegalovirus (CMV), meningitis y traumatismos del hueso temporal. Sin embargo, en más del $50 \%$ de los casos no se identifica la causa a pesar de llevar a cabo un estudio completo (Lin et al., 2017: 912-919).

La HU sin restos auditivos suele ser estable a lo largo del tiempo, al contrario que la HU con restos auditivos y la $\mathrm{HA}$, en las que es frecuente encontrar casos con evolución progresiva o fluctuante, especialmente en la asimétrica en más del $40 \%$ de los casos.

En cuanto al momento de aparición de la hipoacusia, suele ser congénita en el $42 \%$ de las unilaterales y en un $22 \%$ de las asimétricas. En los casos en que estas sorderas no están presentes en el momento del nacimiento, la mayoría suele desarrollarse antes de los 10 años de edad (Lin et al., 2017: 912-919).

En la actualidad, se comienza a comprender los efectos de la HU o HA en el desarrollo infantil y los mecanismos fisiopatológicos involucrados que incluyen el nuevo término "ambliaudia", utilizado para definir el déficit auditivo unilateral que permanece, aun habiendo sido tratado, debido a que la corrección no se ha implementado dentro de la ventana temporal crítica. Este término tiene un paralelismo con la ambliopía en oftalmología (Kaplan et al., 2016: 247-255).

Al considerar tradicionalmente que un solo oído normal es suficiente para el desarrollo típico del habla y del lenguaje del niño, la actitud más frecuente ha sido la de no tratar estos casos y no ofrecer dispositivos 
de asistencia a la audición. Sin embargo, la atención temprana y determinadas tecnologías como los audífonos, la amplificación por vía ósea, el implante coclear, junto con productos de apoyo como sistemas de frecuencia modulada, bucle magnético u otros tipos de conectividad, pueden suponer una ayuda para obtener unos resultados más favorables.

A pesar de que los programas de detección precoz de la sordera detectan las HU o HA de forma eficaz, el manejo de este problema no está estandarizado, por lo que la CODEPEH considera necesario revisar las bases científicas de este trastorno auditivo con el fin de ofrecer un esquema diagnóstico, una actitud clínica ante el problema y un tratamiento uniforme basado en la evidencia.

\section{Impacto sobre el desarrollo del niño}

Hasta hace poco la opinión más frecuente solía ser que la pérdida auditiva unilateral no tenía un impacto significativo sobre el desarrollo del niño. Sin embargo, ya en 1984, Bess y Tharpe fueron los primeros en informar de una consecuencia negativa de la $\mathrm{HU}$ en niños, mostrando que el $35 \%$ de los niños con HU repitieron al menos un año escolar en comparación con el 3,5\% del total de su distrito escolar (Bess y Tharpe, 1984: 206-216).

Varios años después, Oyler y Matkin confirmaron estos hallazgos, mostrando que el $24 \%$ de los niños con HU repitieron un curso comparados con el $2 \%$ en general (Oyler y Matkin, 1987: 27-33). La evidencia de que esta alteración afecta negativamente al desarrollo del niño continuó aumentando con Brookhouser et al. quienes registraron que el $59 \%$ de los niños con HU tenían algún tipo de problema académico o de comportamiento en la escuela (Brookhouser et al., 1991: 1264-1272). Borg et al. informaron que los niños en edad preescolar con esta afección presentaban una alteración del desarrollo del lenguaje (Borg et al., 2002: 308-320). Más recientemente, Lieu et al. demostraron que los niños con HU tenían cuatro veces más probabilidades de haber precisado apoyo educativo y dos veces más de haber recibido logopedia que sus semejantes con audición normal, quedando pues patente el efecto negativo de la HU (Lieu et al., 2010: 1348-1355) (Vila et al., 2015: 271-278) (Krishnan y Van Hyfte, 2016: 63-73) (Fitzpatrick et al., 2017: 1-10).

Aun así muchos profesionales no dan la importancia que se merece a la $\mathrm{HU}$, tal como confirma un estudio realizado a padres de niños con este tipo de pérdida de audición, que concluye que la percepción de estos padres era que los profesionales minimizaban la importancia de esta pérdida auditiva. Asimismo, los padres expresaban su preocupación por el impacto potencial de la pérdida auditiva en el desarrollo de sus hijos (Fitzpatrick et al., 2016: 34-43).

Existen factores individuales de riesgo como el grado de pérdida auditiva y el lado del oído afectado. En cuanto al grado de pérdida, parece que se obtiene peor rendimiento auditivo cuanto mayor es ésta, pero los estudios son muy heterogéneos y no se pueden establecer conclusiones. Casi todos los estudios se realizan en niños con HU grave-profunda y parece que niños con sordera leve-moderada tienen mejores resultados que otros con sordera más grave. Respecto al lado del oído afecto, si se trata del oído derecho, es más 
probable que los niños presenten déficits académicos y de lenguaje, dado que los centros cerebrales del lenguaje reciben su información de ese lado. Hay varios estudios que apoyan esta afirmación. Los niños con HU del oído derecho tienen menores puntuaciones en los test verbales, peor resultado académico, más probabilidad de repetir curso y más probabilidad de retrasos en el lenguaje. Además también se comportan peor en ambientes reverberantes. Otros factores importantes son el estatus socio-económico y el nivel educativo materno (Krishnan y Van Hyfte, 2016: 63-73).

\subsection{Impacto en el reconocimiento del habla}

Para entender el impacto de la pérdida unilateral es preciso aclarar las ventajas que tiene la audición bilateral. La combinación de la suma binaural, el manejo binaural del enmascaramiento y el fenómeno del efecto de la sombra de la cabeza contribuyen a la mejora de la audición y la comprensión de la que disfrutan las personas con audición bilateral normal (Lieu, 2015: 1011-1026).

\section{- Suma binaural}

La suma binaural se refiere a la mayor sensibilidad al sonido por parte del oyente cuando los dos oídos funcionan simultáneamente, en lugar de uno solo, mejorando el procesamiento auditivo central. Los datos experimentales muestran que esta suma es de 2 a 3 dB por encima del umbral, cuando los sonidos son apenas detectables, y puede aumentar hasta $10 \mathrm{~dB}$ cuando los sonidos alcanzan los $90 \mathrm{~dB}$. Debido a que la discriminación del habla mejora con una tasa del $6 \%$ por dB, 2 a 3 dB pueden aportar una mejoría importante en la discriminación del habla (del $12 \%$ al 18\%). Por ello, es evidente que muchas personas se benefician de los audífonos bilaterales o implantes bilaterales, y la suma binaural puede contribuir a esta mejora adicional.

\section{- Enmascaramiento}

El manejo binaural del enmascaramiento o squelch permite entender el lenguaje hablado en medio del ruido de fondo o la conversación. Para una señal de tono puro a $500 \mathrm{~Hz}$, la ventaja de oír por ambos oídos sobre la audición unilateral es de 12 a 15 dB. Para las señales de voz la ventaja aumenta de 3 a 8 dB.

El aumento en la relación señal/ruido (SNR) de 3 dB mejora las puntuaciones de reconocimiento de la palabra alrededor del $18 \%$. Algunos estudios empíricos han documentado que la audición binaural beneficia la inteligibilidad del habla particularmente cuando hay múltiples interferencias sonoras en diferentes lugares alrededor del oyente.

\section{- Efecto de sombra de la cabeza}

El efecto de sombra de la cabeza se refiere a la atenuación del sonido por efecto de la cabeza que se sitúa entre las dos orejas. Empíricamente, hay una reducción de 6,4 dB de la intensidad del habla desde un lado de la cabeza al otro. Para los tonos puros, el efecto de sombra de la cabeza es mayor para las altas frecuencias (20 dB para 5.000 a $6.000 \mathrm{~Hz}$ ). Los niños con HU a menudo describen situaciones sociales incómodas por mala comprensión del mensaje cuando les hablan desde el lado del peor oído. A menos que desarrollen comportamientos falsamente compensatorios para enfrentarse a esta situación, puede parecer que no prestan atención o que ignoran a los otros. Los niños con HU necesitan una SNR mayor de 2 dB dirigida al oído sano para equipararse a las personas con audición normal. 
El efecto de la sombra acústica de la cabeza puede contribuir a la pérdida de percepción de los fonemas consonánticos de alta frecuencia, que son importantes para el reconocimiento del habla (especialmente cuando el ruido se dirige al oído sano y la señal al oído con hipoacusia).

La evidencia indica claramente que los niños y adultos con HU rinden peor que los normoyentes en el reconocimiento del habla en ambientes ruidosos. Requieren una mayor SNR que los normoyentes y son conscientes de ello (Lieu, 2015: 1011-1026).

\subsection{Localización}

Los niños con HU tienen dificultad para identificar la fuente de sonido y cometen más errores en las pruebas de localización del mismo. Este déficit puede dar lugar a situaciones de riesgo en la vida cotidiana al disminuir la capacidad de alerta.

La localización del sonido en el plano horizontal depende del tiempo y el nivel interaural (o intensidad) y es mucho más fácil de lograr con dos oídos que con uno. Los sonidos de baja frecuencia se localizan por diferencias de tiempo interaurales, mientras que los de alta frecuencia se localizan por diferencias de nivel interaurales. La evidencia indica claramente que los niños con HU tienen menos habilidad para la localización del sonido, siendo conscientes de ello (Krishnan y Van Hyfte, 2016: 63-73).

\subsection{Rendimiento académico}

Los logros académicos se pueden medir en función de varios factores, entre los que se incluyen: la repetición de algún curso, la necesidad de apoyo especial y los informes de los profesores. Varios estudios han demostrado que entre un 22-40\% de niños con HU han repetido un curso (comparado con un 2-4\% de los normoyentes). La proporción de niños que necesitan un apoyo individualizado también es mayor (4,4 veces más).

Aunque la mayoría de los estudios se centran en niños con HU grave-profunda, esta diferencia también se observa en pérdidas auditivas de cualquier grado, objetivándose un $59 \%$ de niños con HU que tuvieron problemas académicos y de comportamiento. La evidencia muestra que entre el 22 y el $59 \%$ de niños con HU experimentan dificultades académicas y de comportamiento, presentando mayor fatiga debido al esfuerzo cognitivo adicional dedicado a detectar, decodificar, procesar y comprender (Lieu, 2015: 1011-1026).

\subsection{Capacidad de habla y lenguaje}

Históricamente, se creía que los niños con HU no tenían problemas de habla y lenguaje gracias a la compensación por parte del oído sano. Se ha demostrado que esto no es cierto, puesto que presentan dificultades en el habla, la habilidad oral y de lectura, el deletreo y las escalas de reconocimiento de palabras y de lenguaje.

Una revisión reciente concluye que la mayoría de los estudios informan de peores resultados en los test que valoran el desarrollo del lenguaje en los casos con HU severa y profunda. Estos efectos podrían ser miti- 
gados por los mecanismos de apoyo educativo y un favorable entorno familiar (Anne et al., 2017: 572-579) (Appachi et al., 2017: 565-571).

El $21 \%$ de niños con HU menores de nueve meses demuestran retrasos significativos del comportamiento auditivo precoz y hasta un $41 \%$ tiene retrasos en la vocalización preverbal comparados con normoyentes y en ausencia de otros factores de riesgo auditivo. Lactantes y niños pequeños requieren una mayor SNR que los adultos para comprender los sonidos del habla en presencia de un ruido de enmascaramiento. Así, los niños pequeños con HU pueden experimentar más dificultad con el habla en ambiente ruidoso que los adultos con la misma afección y ciertamente más dificultad que sus compañeros. Por otra parte, varios estudios han demostrado que cuanto mayor es la pérdida auditiva, peor es el habla. El grado de dificultad depende de la edad del niño, de la edad de debut y de la duración de la HU.

Los niños con HU son más propensos a necesitar apoyo logopédico (un $41 \%$ en comparación con el $5 \%$ de normoyentes, y hasta en el doble de casos que sus hermanos normoyentes). A pesar de que existe evidencia de que el lenguaje mejora a lo largo del tiempo, adolescentes con HU continúan con peores puntuaciones que sus hermanos en los test de lenguaje (Krishnan y Van Hyfte, 2016: 63-73).

Por tanto, los niños con esta afección mostraron puntuaciones más bajas en las pruebas de lenguaje receptivo y expresivo en comparación con los niños con normoaudición. Sin embargo, obtuvieron mejores puntuaciones en pruebas de lenguaje expresivo que niños con pérdida auditiva bilateral (José et al., 2014: 198-203).

Entre un 25 y un $40 \%$ de niños con $\mathrm{HU}$ tienen riesgo de desarrollar peores aptitudes en habla y lenguaje comparados con los normoyentes, aunque puedan mejorar parcialmente con la edad.

\subsection{Comportamiento y desarrollo social}

Durante años se ha reconocido que los adultos con HU experimentan una reducción de la calidad de vida social y emocional, puesto que la HU supone una perturbación en la relación con los demás. La HU afecta a la interacción social, prefiriendo la relación de uno en uno, y añade dificultad en las conversaciones (simulan que han oído y entendido lo que se dice, se deben concentrar mucho para entender o entienden mal algunas palabras) (Lieu, 2015: 1011-1026).

Según registros realizados por profesores, un 20-30\% de niños con HU tenían problemas de comportamiento como, por ejemplo, déficit de atención, distracción, retraimiento social, falta de cooperación o agresividad. Al menos un tercio se avergonzaba por su pérdida auditiva y se sentía inferior al resto de compañeros. Los niños que repetían un curso tenían una incidencia mayor de problemas de comportamiento. También obtenían menores puntuaciones en test de medida de calidad de vida, comparados con normoyentes o incluso con otros casos de sordera bilateral. Este tipo de trastornos emocionales debidos a las dificultades en la audición, continuaban en la edad adulta, al realizar cuestionarios similares (desventajas en la audición, sentimientos de frustración, vergüenza, molestia, confusión y falta de ayuda).

En un reciente meta-análisis se ha publicado que niños afectos de HU presentaban puntuaciones más bajas en escalas de calidad de vida, especialmente en el ámbito escolar (Roland et al., 2016: 208-219). 
En general, un tercio de niños con HU presenta problemas en el ámbito del comportamiento social y en las respuestas emocionales a causa de su deficiencia. Éstas continúan incluso en la edad adulta (Krishnan y Van Hyfte, 2016: 63-73).

\subsection{Funciones cognitivas}

Pocos estudios han investigado las funciones cognitivas de niños con HU, aislando correctamente todas las variables y utilizando el cociente de inteligencia $(\mathrm{Cl})$ verbal y no-verbal. Los resultados son diversos, registrándose en algunos casos menor $\mathrm{Cl}$ y en otros no (excepto si habían repetido curso). Las puntuaciones de adolescentes en escalas de $\mathrm{Cl}$ completas eran menores. También los niños con HU han mostrado déficits en la memoria verbal (Krishnan y Van Hyfte, 2016: 63-73). Un meta-análisis reciente concluye que los niños con pérdida auditiva unilateral tienen puntuaciones más bajas en $\mathrm{Cl}$ a escala completa y en el $\mathrm{Cl}$ de rendimiento que los niños con audición normal. También puede haber disparidad en las puntuaciones del Cl verbal (Purcell et al., 2016a: 746-754).

\subsection{Ambliaudia}

La ambliaudia es un término utilizado para describir las dificultades auditivas persistentes en individuos con antecedente de HU durante el período crítico de desarrollo cerebral (Kaplan et al., 2016: 247-255).

El sistema auditivo depende de la integración de señales aferentes provenientes de los dos oídos. Como ya se ha citado, la audición binaural aporta dos grandes ventajas. Por un lado, la redundancia de entradas auditivas lleva a un efecto de suma, que permite disminuir el umbral individual para la detección de sonidos. Por otro lado, las regiones auditivas cerebrales utilizan señales bilaterales, como la diferencia de tiempo interaural y la diferencia de nivel interaural, para aislar señales en ambientes complejos con reverberación y transformar la señal neural unidimensional en señales tridimensionales con representación espacial. Un desequilibrio en la señal auditiva bilateral durante el periodo de desarrollo puede interrumpir la integración de señales binaurales a nivel de la corteza cerebral y centros auditivos; esto se denomina ambliaudia. Estas señales desequilibradas entre los dos oídos durante períodos críticos pueden producir patrones alterados de conexiones neuronales que persisten más allá de la resolución de la patología periférica.

La audición se "aprende". Mientras la cóclea es ya madura desde la semana 23 de la gestación, el desarrollo del proceso de audición y percepción requiere una habilidad auditiva binaural, que necesita casi una década para producirse. Es la única manera de que elementos como la redundancia y la sombra de la cabeza, la supresión y el enmascaramiento (el efecto "cocktail party") entren en juego para permitir la localización del sonido, la percepción del habla sobre el ruido ambiente y la audición espacial (Rohlfs et al., 2017: 475-486). Al ser un fenómeno de reciente descripción, su presentación clínica e impacto a largo plazo aún no están correctamente definidos. Los individuos con riesgo de ambliaudia presentan una hipoacusia con tres aspectos concretos (Kaplan et al., 2016: 247-255):

- La sordera debe ser asimétrica. 
- Debe haber una pérdida de audición medible (habitualmente $>30 \mathrm{~dB}$ ).

- La pérdida de audición debe darse durante el período crítico del desarrollo, en la infancia precoz, con posterior corrección.

En lo relativo al sistema auditivo es bien sabido que la exposición temprana al sonido promueve el desarrollo adecuado y la maduración de los centros de procesamiento auditivo. La plasticidad del sistema auditivo en desarrollo actúa como un mecanismo de adaptación que puede, por ejemplo, ajustarse a las variaciones en el tamaño de la cabeza. Durante los períodos de crecimiento de la cabeza, el ajuste de las relaciones interaurales debe adaptarse continuamente al tiempo que la distancia entre los oídos externos cambia. El hecho de que la experiencia guíe los patrones de la conectividad cerebral se hace evidente cuando la entrada sensorial no es normal, como en los casos de HU.

La presentación clínica de la ambliaudia puede ser sutil y no detectarse en los test audiométricos habituales. Generalmente ocasiona problemas con el proceso auditivo que depende de las señales bilaterales, es decir, la localización del sonido y la supresión de la señal del ruido (dificultad para la comprensión del habla en ambientes ruidosos).

Todos los profesionales involucrados en la atención a niños sordos deben estar al tanto de los datos emergentes acerca de la ambliaudia como entidad diagnóstica y ser conscientes del potencial de consecuencias a largo plazo de la HU y la HA (Kaplan et al., 2016: 247-255).

\subsection{Reorganización cerebral}

Los estudios de resonancia magnética funcional (IRMf) han demostrado una reorganización cortical en los niños con HU que afecta al desarrollo de las redes neuronales para el proceso de señales sensitivas cruzadas. Más aún, esta reorganización parece ser diferente si la pérdida es del oído derecho o del izquierdo, objetivándose disminución del volumen de la sustancia gris y la actividad basal en ciertas áreas cerebrales si la pérdida es derecha (Rohlfs et al., 2017: 475-486).

Por su parte, la HU izquierda mostró cambios más significativos de conectividad que la derecha. Estos resultados indican que la corteza auditiva primaria izquierda en los casos con la HU izquierda ha sido reorganizada por la zona visual y la sensoriomotora a través de la plasticidad intermodal. Además, la reorganización intermodal también altera las redes funcionales cerebrales direccionales. La privación auditiva del lado izquierdo o derecho genera por tanto diferentes efectos en el cerebro humano (Zhang et al., 2016: 149-161).

Existen también diferencias en la integridad microestructural de la sustancia blanca cerebral entre niños con HU y normoyentes. Además, el $67 \%$ de niños con HU causada por un déficit nervioso coclear también asocian anomalías oftalmológicas. Por eso es importante su estudio en estos casos.

Si se produce en períodos tempranos del desarrollo sensitivo, la preferencia aural es difícil de revertir. La deprivación auditiva y la estimulación específica pueden conducir a la reorganización del sistema auditivo central a través de la plasticidad intermodal, especialmente de la corteza auditiva y de las redes funcionales direccionales (Zhang et al., 2016: 149-161). 
En la IRMf, los niños con HU muestran diferencias significativas en cómo se procesa el sonido en la corteza en comparación con los niños con audición normal. En base a que los niños afectados de HU activan las redes de atención con menos fuerza que los niños con audición normal en respuesta a los estímulos auditivos, se podrían explicar los defectos de la memoria, el aprendizaje y los déficit de atención observados en esta población (Vila et al., 2015: 271-278).

Los niños con HU demuestran múltiples diferencias de conectividad funcional entre las redes cerebrales involucradas con la función ejecutiva, la cognición y la comprensión del lenguaje que pueden representar cambios adaptativos, así como maladaptativos. Estos hallazgos sugieren que en algunos niños pueden ser necesarias otras intervenciones o habilitación, más allá de la amplificación, por ejemplo, en forma de ayuda adicional en la escuela (Jung et al., 2017: 2636-2645).

\subsection{Alteraciones del equilibrio}

Existen estudios que concluyen que los niños con HU muestran peores habilidades de equilibrio que los niños normoyentes. Las diferencias significativas entre los dos grupos sólo se observaron en las tareas más difíciles y por lo tanto pueden no detectarse en la evaluación clínica de rutina. Los niños con HU parecen confiar más en la visión para mantener el control postural que sus compañeros normoyentes. Estos hallazgos pueden apuntar a un déficit no sólo de la porción auditiva, sino también de la porción vestibular del oído interno (Wolter et al., 2016: 1589-1595).

\section{Etiología y diagnóstico de la hipoacusia unilateral o asimétrica}

\subsection{Etiología}

La causa más frecuente de las hipoacusias bilaterales es la genética. Sin embargo, en las unilaterales no se da con la misma incidencia. Aun existiendo familias con $\mathrm{HU}$, en un gran número de ellas no se ha conseguido demostrar una mutación genética. También existen síndromes que inicialmente se pueden manifestar con HU, como el braquio-oto-renal o el síndrome de Waardenburg, que avanzarán hacia una hipoacusia bilateral.

En la HU la causa es desconocida entre el $35 \%$ y el $60 \%$ de los casos. La hipoacusia se hace progresiva entre un $14 \%$ y un $32,8 \%$ de los casos, lo cual frecuentemente se asocia con malformaciones en el oído interno. La presencia de alteraciones como un acueducto dilatado es del $31 \%$ al $44 \%$, pudiendo ser en muchos casos bilateral. Otras anormalidades del hueso temporal, como la aplasia o hipoplasia del nervio coclear, es superior en la HU que en la bilateral. En las sorderas severas y profundas unilaterales la aplasia o hipoplasia del nervio coclear es del 50 \% (Clemmens et al., 2013: 318-325) (Nakano et al., 2013: 554-558). Es posible encontrar otras anomalías del hueso temporal como la malformación de Mondini, una cavidad común u otras malformaciones de la cóclea o del vestíbulo.

En niños con hidrocefalia se encuentra un $83 \%$ de casos con pérdida de audición en las frecuencias agudas. 
Las causas más frecuentes de inicio postnatal de la HU son la infección congénita por citomegalovirus (CMV), los traumatismos craneales y la meningitis. Se ha visto que 2/3 de los infectados por CMV asintomáticos debutarán con una HU (Rohlfs et al., 2017: 475-486).

Actualmente, gracias a la aplicación de los calendarios de vacunación, es poco frecuente encontrar sorderas secundarias a paperas, sarampión o rubeola, aunque las corrientes antivacunas incrementan el riesgo de aparición de estas antiguas enfermedades. Por ello, se debe comprobar siempre la correcta vacunación de los niños con hipoacusia neurosensorial.

En la etiología de la HU no se deben olvidar los problemas del oído medio como la atresia auris unilateral, el colesteatoma, la otitis media crónica, la otosclerosis, las discontinuidades o las malformaciones de la cadena osicular (Lieu, 2015: 1011-1026).

Respecto a la etiología de la HA, aun pudiendo coincidir la noxa común a las unilaterales, la frecuencia varía, siendo más frecuentes otras etiologías.

En la HA son más frecuentes las mutaciones genéticas: $56,8 \%$ de los casos frente al $28 \%$ de las unilaterales, siendo la mutación más frecuente la GJB2. Las hipoacusias progresivas o fluctuantes son más frecuentes en hipoacusias asimétricas que en las unilaterales (Lin et al., 2017: 912-919).

\subsection{Diagnóstico}

Aunque la incidencia de las noxas es diferente en la HU y en la bilateral asimétrica, para su diagnóstico se deben seguir las mismas pautas en ambos procesos. Este tema ha sido ya tratado en un anterior documento de recomendaciones de la CODEPEH (Núñez et al., 2015), coincidente con las últimas publicaciones al respecto (Van Beeck et al., 2017: 185-191) (Gruber et al., 2017: 688-695).

\section{Tratamiento de la hipoacusia unilateral o asimétrica}

Si bien no existe un protocolo único para todas las situaciones, hay una variedad de opciones disponibles para los niños con una HU que van desde la modificación de su ubicación en el aula, la adaptación de audífonos, de dispositivos CROS (contralateral routing of signal), de sistemas osteointegrados para la estimulación por vía ósea y de implantes cocleares, así como el uso de productos de apoyo, tales como los sistemas de frecuencia modulada. También se aconseja evitar la exposición a ruidos intensos y vigilar la presencia de otitis media secretora que pudiera afectar la audición del oído con audición normal. Como cualquier otra intervención, ha de ser individualizada teniendo en cuenta las necesidades de cada niño y su familia, así como sus expectativas (Krishnan y Van Hyfte, 2016: 63-73).

En base a lo anteriormente expuesto, y a las indicaciones de Purcell et al. (2016b: 43-48), se propone el algoritmo que refleja la figura 1. 


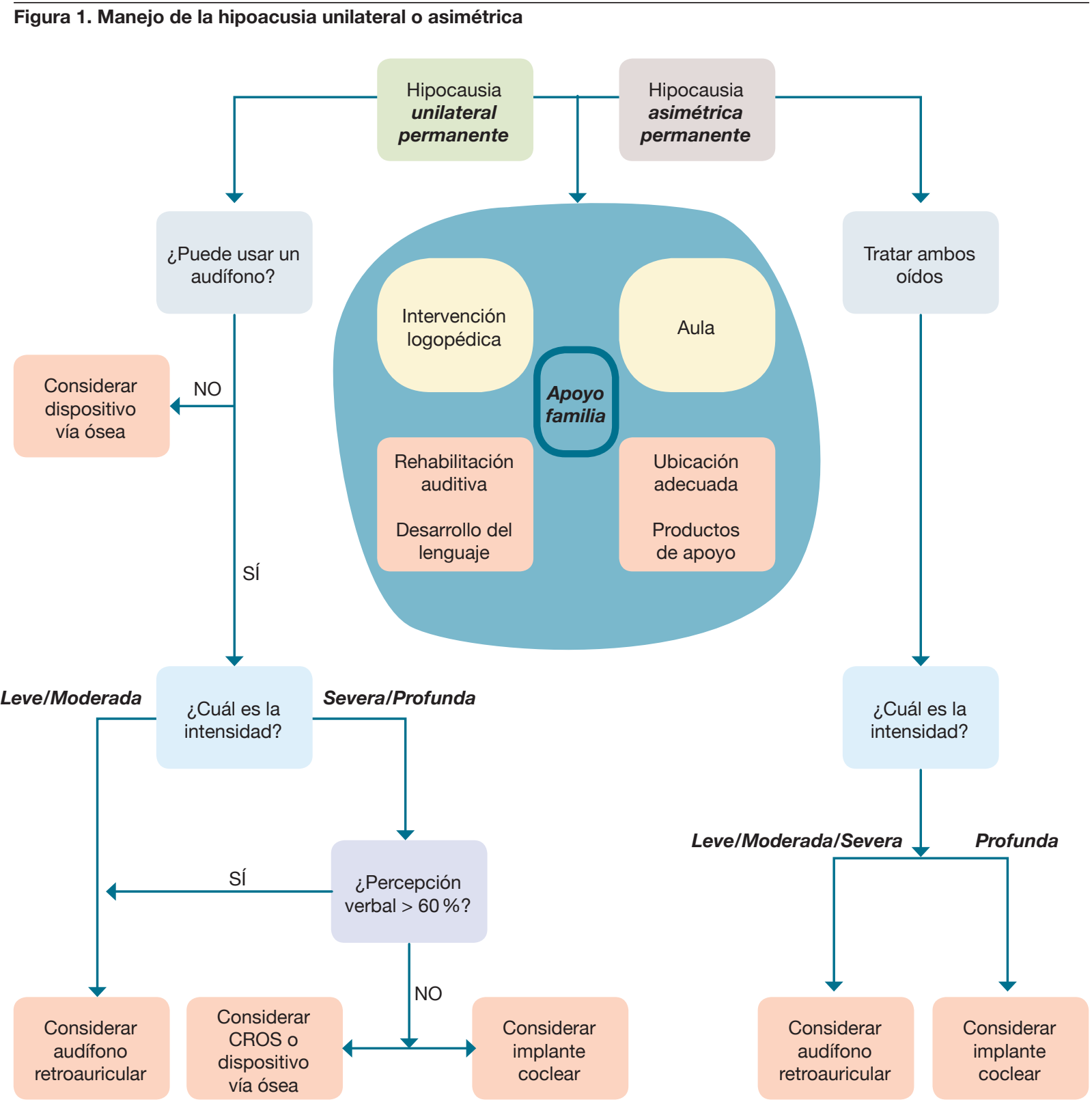

Fuente: elaboración propia.

\subsection{Audífonos convencionales}

Los audífonos pueden representar una opción viable de intervención para los niños con HU que conservan restos auditivos útiles en el oído afectado. No obstante, los clínicos no recurren a esta medida de forma consistente. Solamente se recomienda de forma inicial la adaptación audioprotésica al $26 \%$ de los niños 
con HU (Fitzpatrick et al., 2014: 10-18), cifrándose entre el 9-48\% el porcentaje de niños adaptados (Davis et al., 2001: 179-186).

Una encuesta realizada a padres indica similares resultados: el $42 \%$ de las familias de niños con HU fueron informadas de que los audífonos podrían no ser de ayuda, aunque solamente el $8 \%$ de los niños no contaban con una audición residual (Krishnan y Van Hyfte, 2016: 63-73). Sin embargo, existen evidencias de que un audífono convencional aporta beneficios: 20 padres de niños con HU moderada encuestados contestaron que observaron una mejor audición y rendimiento académico y social con el audífono, indicando que hubieran deseado una adaptación más temprana. Los niños con adaptación audioprotésica también refieren mayor facilidad de escucha tanto en ambientes silenciosos, como en los ruidosos. Aun sin encontrarse un cambio cuantificable en la percepción verbal, los padres, los maestros y los propios niños adaptados son conscientes del beneficio del uso del audífono en casa y en la escuela, así como en calidad de vida (Briggs et al., 2011: 448-454). Los niños pequeños con HU moderada y severa también muestran una significativa mejoría en la localización de los sonidos, aunque los niños con adaptaciones realizadas a mayor edad muestran interferencia bilateral. De hecho, solamente el $26 \%$ de los niños con HU usan el audífono permanentemente, frente al $72 \%$ de los niños con hipoacusia bilateral moderada. Esta pobre adherencia al uso del audífono en los niños con HU puede ser debida a que frecuentemente se les adapta a mayor edad que a los niños con hipoacusias bilaterales. En niños adaptados tardíamente el uso de los audífonos es menor (Reeve, 2005: 20-21).

En contraste a lo que ocurre con los adultos que pierden de forma aguda uno de sus oídos y son conscientes de la pérdida de la audición binaural, los niños con HU congénita nunca han experimentado dicha audición y, por tanto, no reconocen esta pérdida. La adaptación de un audífono puede exponer al oído afectado a una intensidad excesiva que el cerebro integra con dificultad y ello podría explicar la aversión al uso del audífono. Dado que la plasticidad neural permite alcanzar la integración binaural de las señales auditivas, es necesario un periodo de tiempo para alcanzar la necesaria compensación que se requiere para tolerar y aceptar la adaptación audioprotésica. Aunque el niño desarrolle de forma lenta y progresiva la HU, esta adaptación unilateral precisa de un tiempo de ajuste como en el caso de las hipoacusias bilaterales para que la plasticidad neural actúe (Lieu, 2015: 1011-1026).

El método prescriptivo utilizado por el audioprotesista puede representar una limitación, ya que no existe evidencia de que los niños con una HU prefieran la ganancia obtenida por los métodos usados en las hipoacusias bilaterales. Una posible solución a este problema es utilizar una técnica que consiga una fusión binaural de la HA (McSpaden y Brethower, 2007: 40). Sin embargo, este método requiere la participación del sujeto y, por tanto, no es útil en la adaptación de niños pequeños con $\mathrm{HU}$.

Por tanto, las evidencias disponibles en la actualidad indican que los niños con una HU con restos auditivos útiles en el oído afectado pueden encontrar beneficio en la amplificación convencional digital, que reporta una mayor facilidad de escucha, mejor rendimiento en situaciones sociales y académicas, y mejor localización, si son adaptados precozmente. No obstante, se precisan estudios adicionales en lo que respecta al método prescriptivo más apropiado para los niños con HU (Krishnan y Van Hyfte, 2016: 63-73). 


\subsection{CROS (Contralateral Routing Of Signals)}

Un audífono tipo CROS se basa en colocar un micrófono en el oído hipoacúsico para llevar la señal a un receptor situado en el oído sano. El beneficio se obtiene al restaurar la habilidad de detectar las señales dirigidas hacia el oído afectado. Aunque se encuentra algún beneficio de su uso en ambientes tranquilos, no se considera una buena opción para los niños pequeños y para el entorno de un aula, dado que no existe una mejora significativa del reconocimiento verbal e, incluso, causa efectos negativos en algunos casos. Aunque en adultos algunos estudios muestran que estos sistemas ayudan a mejorar el reconocimiento de la palabra y la localización del sonido, no parece que sea una opción aconsejable para los niños que aún no tienen la habilidad necesaria para manipular su entorno con el fin de asegurar que llegue al oído sano una señal óptima (Krishnan y Van Hyfte, 2016: 63-73) (American Academy of Audiology, 2013).

\subsection{Sistemas osteointegrados}

Esta es otra opción terapéutica en la HU, ya sea por una agenesia del oído medio, por hipoacusias secundarias a cirugía del oído que por diversas causas no permitan adaptar un audífono convencional, por una hipoacusia profunda neurosensorial coclear o por una hipoacusia debida a agenesia de nervio auditivo.

En las atresias y otras hipoacusias de trasmisión se puede conseguir una audición binaural, no así en los casos de sordera neurosensorial ya que estará funcionando como un sistema CROS, llevando la audición del lado afecto al sano.

En los casos de agenesia del nervio auditivo unilateral, ésta será la única opción para tener una sensación auditiva en el oído afectado.

Los estudios han demostrado que los dispositivos de este tipo restauran la sensación de la audición del lado hipoacúsico y generalmente mejoran la satisfacción y la percepción subjetiva del habla en ambiente ruidoso (Christensen et al., 2010: 175-177). Estos dispositivos eliminan, con buenos resultados, el efecto sombra de la cabeza pero no producen beneficios para mejorar la localización del sonido (Monini et al., 2015: 381-388).

Los audífonos de conducción ósea se han convertido en una de las modalidades más importantes en el tratamiento en la HU (Peters et al., 2015: 218-226) (Doshi et al., 2013: 100-103). Todos los dispositivos comparten el mismo mecanismo de conducción del estímulo a través del cráneo desde el lado hipoacúsico a la cóclea del oído con audición normal, sin embargo, difieren en el mecanismo por el cual la señal es transmitida al hueso. Se pueden usar de forma no implantable, mediante una softband, o de forma implantable anclándolos al hueso. Se debe recordar que no permiten la audición del oído afecto y que, aunque sí mejoran las pruebas subjetivas de la percepción del lenguaje, no lo hacen en las objetivas (Dornhoffer y Dornhoffer, 2016: 522-528).

\subsection{Implantes cocleares}

Los criterios para ser candidato a un implante coclear están evolucionando. Los niños con HU o pérdida auditiva asimétrica, que no vienen siendo considerados candidatos a implante coclear, deben ser evaluados 
individualmente. El manejo audiológico de estos casos no es muy diferente en comparación con los niños que tradicionalmente sí son considerados receptores de implantes cocleares. Las técnicas de evaluación y habilitación deben ser modificadas para aislar el oído implantado para obtener resultados precisos y proporcionar una intervención terapéutica significativa (Greaver et al., 2017: 91-98).

La justificación fundamental para realizar un implante coclear en una hipoacusia neurosensorial unilateral de origen coclear es conseguir una audición binaural y conservar la vía auditiva del lado afecto, evitando la reorganización cerebral y sus consecuencias.

El uso de un audífono y un implante de forma simultánea no genera ningún conflicto en la integración auditiva, ni tampoco lo causa utilizar un implante en el oído afectado teniendo en el otro una audición normal (Polonenko et al., 2017: 681-689).

Los estudios realizados en niños con HU neurosensorial congénita que recibieron un implante coclear a edades comprendidas entre los 3, 6 y 11 años tuvieron una moderada pero significativa mejoría en la comprensión del habla, la capacidad de lateralización y el sentido subjetivo de la percepción auditiva.

Otros estudios hacen referencia a la falta de conclusiones firmes que nos puedan indicar la efectividad del implante coclear en niños con $\mathrm{HU}$, debido a la heterogeneidad de hallazgos, a los pequeños tamaños de las muestras y a la falta de estudios de alto nivel de evidencia.

A pesar de ello, la implantación coclear puede ser una opción efectiva de tratamiento en niños con HU (Peters et al., 2016: 713-721). Sin embargo, el limitado número de usuarios y la posibilidad de estigmatización en los niños mayores sugieren que el asesoramiento a los padres sobre la implantación coclear en niños con HU neurosensorial congénita debe ser comprensible, objetivo y crítico con respecto a la escasez de datos publicados hasta ahora (Thomas et al., 2017: 496-503).

Es importante desarrollar criterios adecuados para realizar implantes cocleares en la HU neurosensorial congénita ya que el retraso en la implantación puede producir resultados no óptimos (Boyd, 2015: 121-136).

Hay algunos casos de especial consideración como son las HU neurosensoriales congénitas secundarias al CMV y las malformaciones del oído interno en caso de existir éstas en ambos oídos, dada la alta probabilidad de pérdida del oído sano contralateral. Si esto ocurriese en edades del desarrollo del lenguaje, podría tener un impacto muy importante en la vida del niño.

\subsection{Productos de apoyo en el aula}

Los productos de apoyo como los sistemas de FM, el bucle de inducción magnética u otros tipos de conectividad, transmiten de forma inalámbrica la señal al receptor, con el objeto de mejorar la relación señalruido y reducir los efectos negativos del ruido y de la reverberación, así como de la distancia respecto al interlocutor y/o la fuente sonora.

Los sistemas de FM son el producto de apoyo más frecuentemente utilizado en las aulas. Existen muchos estudios que demuestran que los sistemas FM mejoran el reconocimiento de la palabra en los niños con 
$\mathrm{HU}$, tanto en ambientes tranquilos, como en los ruidosos. La mejoría de la comprensión de la palabra es patente aun recibiendo el oído afectado el ruido directamente, observándose que, a mayor severidad de la HU, mayor beneficio obtenido del uso del sistema FM. Incluso, se ha constatado que los sistemas FM mejoran significativamente la comprensión de la palabra frente a las adaptaciones CROS o el uso de audífonos convencionales solos. En el caso de los niños con HU, la forma de acoplarles el sistema de FM es particularmente importante (Bess y Gravel, 1981: 24-29) (Purcell et al., 2016b: 43-48).

\subsection{Ubicación en el aula}

Además de los dispositivos que pueden ser adaptados al niño con una HU, la ubicación preferente en el aula juega un importante papel ya que puede ayudar a mejorar su audición, siempre que se favorezca con la misma al oído sano. Se sabe que los niños con HU han de ser ubicados a la mitad de la distancia del docente que la de un niño con audición normal para tener la misma discriminación verbal (Noh y Park, 2012: 426-432). La ubicación preferente en el aula y el uso de productos de apoyo debe realizarse con el menor impacto sobre el niño para que no le diferencien del resto de sus compañeros (Purcell et al., 2016b: 43-48).

\section{Calidad de vida}

La literatura científica al uso ha puesto de relieve la importancia de la audición binaural tanto para la propia percepción auditiva, como para el desarrollo del lenguaje y de otras habilidades cognitivas que dependen de éste, así como para el desarrollo global del niño (Rohlfs et al., 2017: 475-486). De acuerdo con lo ya expuesto en los apartados precedentes, en la práctica clínica, y desde el punto de vista educativo y social, una HU o HA puede ser minimizada en sus consecuencias con un precoz y adecuado diagnóstico y tratamiento.

Calidad de vida es un concepto amplio influido de un modo complejo por la salud de la persona, su estado psicológico, su nivel de independencia, sus relaciones sociales, así como por su relación con los elementos esenciales de su entorno. Por ello, y en todo caso, habrá que tener en cuenta la audición como elemento de análisis e influencia sobre esa valoración y percepción que una persona con sordera puede tener acerca de cada una de estas dimensiones.

\section{1 . Factores que influyen en la calidad de vida}

Todo lo expuesto parece evidenciar que un solo oído no es suficiente para el desarrollo adecuado del habla y del lenguaje, para el control motriz y del equilibrio, y para la conducta, generando déficits de aprendizaje, con consecuencias a nivel escolar, y déficits comunicativos, con consecuencias a nivel de interacción e inclusión social. Sin embargo, no siempre estas consecuencias están bien identificadas, ni los apoyos de distinta naturaleza: protésico, logopédico y/o escolar, etc., que se requieren en cada caso, se proporcionan de forma generalizada. 
Algunos de los factores que hay que considerar a la hora de evaluar cómo afecta una HU o HA sobre la calidad de vida, son los que se exponen a continuación, pudiendo afirmar que estas consideraciones serían válidas para cualquier pérdida auditiva.

\section{- Detección y diagnóstico precoz}

Son elementos de gran importancia en la medida en que influyen decisivamente en el mejor pronóstico, bienestar físico, emocional y social del niño, pero también de su familia, a la que reporta en el medio plazo menos costes económicos, sociales y emocionales, si se sigue además el oportuno tratamiento tras dicho diagnóstico. Sin embargo, la ausencia de programas de cribado o los fallos en el mismo pueden retrasar el diagnóstico hasta la edad escolar, ya que en muchos casos los síntomas de la HU o HA son muy sutiles y pueden ser atribuidos a comportamientos circunstanciales o a retrasos evolutivos.

Por ello, será necesario:

- Optimizar la eficacia y los estándares de calidad necesarios en la aplicación del Programa de Detección Precoz de la sordera infantil y el Programa del "Niño sano", favoreciendo la captación de los casos que superen el cribado neonatal.

- Garantizar un control auditivo regular a los casos de HU o HA, dado que en una alta proporción de casos presentan una pérdida auditiva progresiva (Lin et al., 2017: 912-919). Un estudio prospectivo, a lo largo de trece años, sobre una población entera de niños diagnosticados en una región de Canadá (Ontario), mostró como hallazgos clave que más del 40 \% de los niños diagnosticados inicialmente con HU estaban en riesgo de tener mayor deterioro de la audición con posterioridad y que, aproximadamente, uno de cada seis desarrollaría una pérdida auditiva bilateral. Este hallazgo subraya la importancia de la realización de un seguimiento audiológico de los niños con HU y la necesidad de un asesoramiento cuidadoso a las familias para no minimizar las consecuencias de estas pérdidas de audición y poder actuar en consecuencia tras el diagnóstico inicial. De hecho, y según este mismo estudio, se espera que al menos el $20 \%$ de los casos tenga una sordera diferida en el tiempo (Fitzpatrick et al., 2017: 1-10).

\section{- Consecuencias sobre el desarrollo, los aprendizajes, la conducta y las interacciones sociales}

Como se ha dicho, los niños con pérdida auditiva, en general, deben hacer un esfuerzo cognitivo adicional para detectar, decodificar, procesar y comprender el lenguaje, singularmente ante la presencia de nuevas palabras y en el caso de actividades multitarea, cuando deben prestar atención a varias fuentes de estímulos e información. Especialmente importante es esta situación en las primeras edades cuando se está desarrollando el lenguaje y cuando es esencial la comprensión de los sonidos del habla para adquirir la competencia lingüística. Todo ello puede tener consecuencias negativas sobre sus aprendizajes escolares (Lieu, 2015: 1011-1026).

La evaluación motora y del equilibrio son otros dos factores a tener en cuenta para calibrar el impacto de potenciales factores multisensoriales en niños con HU o HA. No hay que perder de vista además que las conductas controladoras del equilibrio se desarrollan en una edad temprana (Wolter et al., 2016: 1589-1595).

Por último, si bien un adulto que adquiere una HU tiene dificultades en su interacción social y ve afectada su calidad de vida (malos entendidos, mayor necesidad de atención y concentración, pérdida de información, 
etc.), también los niños con HU o HA identifican con claridad las dificultades que encuentran motivadas por estas pérdidas de audición cuando no son conscientes de que se les habla desde el lado del oído afectado, cuando no prestan atención cuando se les habla o ignoran una llamada.

De ahí la necesidad de:

- Llevar a cabo una valoración logopédica para determinar la necesidad de una intervención especializada y su intensidad. Es preciso, por ello, un seguimiento de los casos que presentan HU o HA, particularmente si se observan retrasos en el desarrollo del lenguaje, déficits en la adquisición de vocabulario, dificultades en el desarrollo lectoescritor y alteraciones en el habla por déficits/errores fonológicos y gramaticales $u$ otro tipo de alteraciones del lenguaje derivadas de una percepción auditiva deficiente (Núñez et al., 2016).

- Minimizar el impacto de las limitaciones de equilibrio asociadas a la HU o HA a través de la estimulación temprana del control del equilibrio, junto con un control estricto de la salud visual.

- Optimizar la función auditiva, empleando los dispositivos auditivos y tecnológicos más adecuados en cada caso, con provisión de productos de apoyo como los sistemas FM u otro tipo de dispositivos que, en conexión con las prótesis auditivas, acercan la señal y permiten superar las limitaciones impuestas por el ruido ambiente y la reverberación.

- Facilitar los apoyos necesarios en el ámbito educativo para favorecer el desarrollo cognitivo y los aprendizajes, así como la comunicación y la participación.

- Promover la accesibilidad auditiva en las aulas, tanto por lo que respecta a las condiciones de edificación y adecuación de las mismas con el empleo de materiales y mobiliario que reduzca el ruido ambiente y la reverberación, como facilitando al alumno con sordera los productos de apoyo a la audición (sistemas FM, bucle magnético u otros) que, en conexión con sus prótesis auditivas, acerquen y mejoren la señal sonora.

\section{- Respuesta de los diferentes sistemas}

Un factor de gran relevancia es la adecuada y suficiente respuesta de los distintos sistemas implicados (sanitario, educativo y social) en cuanto a identificación, tratamiento (incluidas las prótesis), seguimiento y recursos de apoyo. En un estudio longitudinal (Lieu et al., 2010: 1348-1355), a lo largo de tres años, sobre 49 niños, entre 6-12 años de edad, con HU, se evaluaron tanto aspectos cognitivos como el desarrollo del lenguaje y se encontró que, aproximadamente, el $25 \%$ de los sujetos continuó mostrando dificultad académica después de tres años, a pesar de la mejora progresiva que se apreciaba sobre los resultados obtenidos por los niños en dichos aspectos (Kuppler et al., 2013: 617-622).

Por tanto, además de todo lo ya expresado, será preciso:

- Hacer una evaluación y seguimiento global de la evolución del niño que incluya no solo parámetros de desarrollo del lenguaje, sino también de la conducta, del rendimiento escolar, del comportamiento y las relaciones sociales y de calidad de vida (Lieu, 2015: 1011-1026). 


\section{- Atención y apoyo a las familias}

La existencia de diferentes opciones de tratamiento exige un adecuado nivel de información y formación en los profesionales clínicos, quienes habrán de mantener una actitud proactiva en relación con la información que debe recibir la familia para la toma de decisiones.

Es importante además evitar la desorientación de las familias y/o la demora o la falta de respuesta: pautas, recursos, derivación adecuada, etc., sin perder de vista la situación individual del caso y la variabilidad de las conductas familiares, dado que la calidad de vida no sólo se mide sobre el propio niño y sus progresos, sino sobre su familia.

Por ello, se debe:

- Informar y orientar a padres y educadores en relación con la adopción de determinadas estrategias comunicativas, siendo prioritario en todo caso que el niño se sitúe siempre de la forma más favorable en relación con la fuente de sonido, tanto en las aulas, como en una reunión familiar, en la casa, respecto al televisor, etc., facilitando siempre el acceso visual (Núñez et al., 2016).

- Sensibilizar e informar acerca de la prevención y cuidado del oído funcional y/o con normoaudición, con el fin de preservar la salud auditiva.

La pérdida auditiva neurosensorial unilateral en niños es un déficit común que puede representar un reto no solo médico, sino educativo y social. Tanto la ausencia de diagnóstico precoz, como de un tratamiento eficaz se han mostrado perjudiciales para los niños, especialmente en el entorno escolar (Dornhoffer y Dornhoffer, 2016: 522-528).

El diagnostico precoz, el tratamiento orientado a la restauración de la audición binaural, la provisión de dispositivos de apoyo, la atención temprana que incluya intervención logopédica especializada, la información y formación de los profesionales clínicos y docentes, la orientación y el apoyo a las familias, se revelan claves para la superación de las limitaciones y dificultades que puede ocasionar una HU o HA (Kuppler et al., 2013: 617-622).

\section{Recomendaciones CODEPEH 2017}

Tal como se ha expuesto, la ausencia de tratamiento en la hipoacusia unilateral o asimétrica puede generar problemas que hasta hace poco tiempo no se tenían en cuenta, dando por hecho que la hipoacusia unilateral no interfería de forma importante en la vida de los niños. Sin embargo, se sabe que la hipoacusia unilateral o asimétrica dificulta el reconocimiento del habla en ambientes contaminados por el ruido, alterando el desarrollo del lenguaje, el rendimiento académico y la interacción social. Además, pueden dar lugar a una reorganización de la vía auditiva a nivel cerebral, que condicionaría la efectividad del tratamiento. 
Por tanto, con objeto de evitar y/o paliar consecuencias no deseadas de la hipoacusia unilateral o asimétrica sobre el desarrollo del niño, su conducta y sus relaciones personales y, particularmente, sobre su calidad de vida (bienestar emocional, bienestar físico e inclusión social), la CODEPEH recomienda:

1. Incorporar la hipoacusia unilateral o asimétrica a los protocolos habituales de manejo de las pérdidas auditivas, dada la importante repercusión en el desarrollo integral del niño.

2. Hacer el estudio etiológico correspondiente para orientar el tratamiento más adecuado en cada caso.

3. Mantener una actitud activa y precoz, asesorando a los padres sobre pros y contras de las distintas modalidades de tratamiento para una adecuada toma de decisiones.

4. Identificar correctamente el efecto de la hipoacusia unilateral o asimétrica sobre el niño para determinar qué apoyos de distinta naturaleza: protésicos y tecnológicos, logopédicos y educativos, etc. se requieren en cada caso.

5. Garantizar el control auditivo regular en los casos de hipoacusia unilateral o asimétrica, dado que en una alta proporción de casos presentan una pérdida auditiva progresiva.

6. Informar y orientar a padres y educadores en relación con la adopción de determinadas estrategias comunicativas, así como acerca de la prevención y cuidado del oído funcional y/o con normoaudición.

7. Incluir dentro de las prestaciones sanitarias del Sistema Nacional de Salud la financiación del tratamiento protésico en los casos de las sorderas unilaterales. 
American Academy of Audiology (2013): Clinical practice guidelines: pediatric amplification. Reston, Virginia: American Academy of Audiology.

Anne, S. et al. (2017): "Speech and language Consequences of Unilateral Hearing Loss: A Systematic Review". Otolaryngology-Head \& Neck Surgery, 157 (4): 572-579.

Appachi, S. et al. (2017): "Auditory outcomes with hearing rehabilitation in children with unilateral hearing loss: a systematic review". Otolaryngology-Head \& Neck Surgery, 157 (4): 565-571.

Bess, F. H. y Gravel, J. S. (1981): "Recent trends in educational amplification”. Hear Instrum, 32: 24-29.

Bess, F. H. y Tharpe, A. M. (1984): “Unilateral hearing impairment in children”. Pediatrics, 74 (2): 206-216.

Boyd, P. J. (2015): "Potential benefits from cochlear implantation of children with unilateral hearing loss". Cochlear Implants International, 16 (3): 121-136.

Borg, E. et al. (2002): "Behavioural awareness, interaction and counselling education in audiological rehabilitation: development of methods and application in a pilot study". International Journal of Audiology, 41 (5): 308-320.

Briggs, L. et al. (2011): "Outcomes of conventional amplification for pediatric unilateral hearing loss". Annals of Otology, Rhinology and Laryngology, 120 (7): 448-454.

Brookhouser, P. E. et al. (1991): “Unilateral hearing loss in children”. The Laryngoscope, 101 (12): 1264-1272.

Clemmens, C. S. et al. (2013): “Unilateral cochlear nerve deficiency in children”. Otolaryngology-Head \&Neck Surgery, 149 (2): 318-325.

Christensen, L. et al. (2010): "Update on bone-anchored hearing aids in pediatric patients with profound unilateral sensorineural hearing loss". Otolaryngology-Head \& Neck Surgery, 136 (2): 175-177.

Davis, A. et al. (2001): "Children with mild and unilateral hearing loss", en Seewald, R.C. y Gravel, J.S. (eds.): $A$ Sound Foundation through Early Amplification: Proceedings of the Second International Conference. Stäfa (Suiza): Phonak AG.

Dornhoffer, J. R. y Dornhoffer, J. L. (2016). "Pediatric unilateral sensorineural hearing loss: implications and management". Current Opinion in Otolaryngology \& Head and Neck Surgery, 24 (6): 522-528.

Doshi, J. et al. (2013): "Quality-of-life outcomes after bone-anchored hearing device surgery in children with singlesided sensorineural deafness". Otology \& Neurotology, 34 (1): 100-103.

Fitzpatrick, E. et al. (2014): "Mild bilateral and unilateral hearing loss in childhood: a 20-year view of hearing characteristics, and audiologic practices before and after newborn hearing screening". Ear and Hearing, 35 (1): 10-18.

Fitzpatrick, E. et al. (2016): "Children with Mild Bilateral and Unilateral Hearing Loss: Parents' Reflections on Experiences and Outcomes". Journal of Deaf Studies and Deaf Education, 21 (1): 34-43.

Fitzpatrick, E. et al. (2017): "Characteristics of children with unilateral hearing loss". International Journal of Audiology, 56 (11): 819-828.

Greaver, L. et al. (2017): "Considerations for Pediatric Cochlear Implant Recipients With Unilateral or Asymmetric Hearing Loss: Assessment, Device Fitting, and Habilitation”. American Journal of Audiology, 26: 91-98. 
Gruber, M. et al. (2017): "Concomitant imaging and genetic finding in children with unilateral sensorioneural hearing loss". The Journal of Laryngology \& Otology, 131 (8): 688-695.

José, M. R. et al. (2014): "Language disorders in children with unilateral hearing loss: a systematic review". International Archives of Otorhinolaryngology, 18 (2): 198-203.

Jung, M. E. et al. (2017): "Differences in interregional brain connectivity in children with unilateral hearing loss". The Laryngoscope, 127: 2636-2645.

Kaplan, A. B. et al. (2016): "Amblyaudia: review of pathophysiology, clinical presentation, and treatment of a new diagnosis". Otolaryngology-Head \& Neck Surgery, 154 (2): 247-255.

Krishnan, L. A. y Van Hyfte, S. (2016): "Management of unilateral hearing loss". International Journal of Pediatric Otorhinolaryngology, 88: 63-73.

Kuppler, K. et al. (2013): "A review of unilateral hearing loss and academic performance: Is it time to reassess traditional dogmata?". International Journal of Pediatric Otorhinolaryngology, 77 (5): 617-622.

Lieu, J. E. et al. (2010): "Unilateral hearing loss is associated with worse speech-language scores in children". Pediatrics, 125 (6): 1348-1355.

Lieu, J. E. (2015): “Management of children with unilateral hearing loss”. Otolaryngology Clinics, 48 (6): 1011-1026.

Lin, P. H. et al. (2017): "Etiologic and audiologic characteristics of patients with pediatric-onset unilateral and asymmetric sensorineural hearing loss". JAMA Otolaryngology \& Head and Neck Surgery, 143 (9): 912-919.

May, J. J. (2000): “Occupational hearing loss”. American Journal of Industrial Medicine, 37 (1): 112-120.

McSpaden, J. B. y Brethower, L. D. (2007): “Achieving binaural fusion in asymmetric losses”. The Hearing Review, 14: 40.

Monini, S. et al. (2015): "Bone conductive implants in single-sided deafness". Acta Otolaryngologica, 135 (4): 381 388.

Nakano, A. et al. (2013): "Cochlear nerve deficiency and associated clinical features in patients with bilateral and unilateral hearing loss”. Otology \& Neurotology, 34 (3): 554-558.

Noh, H. y Park, Y. (2012): "How close should a student with unilateral hearing loss stay to a teacher in a noisy classroom?" International Journal of Audiology, 51 (6): 426-432.

Núñez, F. et al. (2015): "Diagnóstico etiológico de la sordera infantil: recomendaciones CODEPEH 2015”. 2a ed. Revista FIAPAS, 155, Separata.

Núñez, F. et al. (2016): "Diagnóstico y tratamiento de la otitis media secretora infantil: recomendaciones CODEPEH 2016". $2^{\mathrm{a}}$ ed. Revista FIAPAS, 159, Separata.

Oyler, R. F. y Matkin, N. D. (1987): "National survey of educational preparation in pediatric audiology". ASHA, 29 (1): 27-33.

Peters, J. P. et al. (2015): "Review: bone conduction devices and contralateral routing of sound systems in singlesided deafness". The Laryngoscope, 125 (1): 218-226.

Peters, J. P. et al. (2016): "Cochlear implantation in children with unilateral hearing loss: a systematic review". The Laryngoscope, 126 (3): 713-721. 
Polonenko, M. J. et al. (2017): "Children with single-sided deafness use their cochlear implant”. Ear and Hearing, 38 (6): 681-689.

Purcell, P. L. et al. (2016a): "Children with unilateral hearing loss may have lower intelligence quotient scores: A meta-analysis". The Laryngoscope, 126 (3): 746-754.

Purcell, P. L. et al. (2016b): "Hearing devices for children with unilateral hearing loss: patient- and parent-reported perspectives". International journal of pediatric otorhinolaryngology, 90: 43-48.

Reeve, K. (2005): "Audiological management and family factors for children with mild and uni-lateral hearing impairment", en Centers for Disease Control and Prevention: National Workshop on Mild and Unilateral Hearing Loss: Workshop Proceedings. Breckenridge, Colorado: Centers for Disease Control and Prevention.

Rohlfs, A. K. et al. (2017): "Unilateral hearing loss in children: a retrospective study and a review of the current literature". European Journal of Pediatrics, 176 (4): 475-486.

Roland, L. et al. (2016): "Quality of life in children with hearing impairment: systematic review and meta-analysis". Otolaryngology \& Head and Neck Surgery, 155 (2): 208-229.

Ross, D. S. et al. (2010): "Highly variable population-based prevalence rates of unilateral hearing loss after the application of common case definitions". Ear and Hearing, 31 (1): 126-133.

Thomas, J. P. et al. (2017): "Cochlear implantation in children with congenital single-sided deafness". Otology \& Neurotology, 38 (4): 496-503.

Van Beeck, E. A. et al. (2017): "High prevalence of abnormalities on CT an MR imaging in children with unilateral sensorineural hearing loss irrespective of age or degree of hearing loss". International journal of pediatric otorhinolaryngology, 97: 185-191.

Vila, P. et al. (2015): "Asymmetric and unilateral hearing loss in children”. Cell and Tissue Research, 361 (1): $271-$ 278.

Wolter, N. E. et al. (2016): "Unilateral hearing loss is associated with impaired balance in children: a pilot study". Otology \& Neurotology, 37 (10): 1589-1595.

Zhang, G. Y. et al. (2016): "Changes of the directional brain networks related with brain plasticity in patients with long-term unilateral sensorineural hearing loss". Neuroscience, 313: 149-161. 